\title{
Charge exchange spectroscopy as a fast ion diagnostic on TEXTOR
}

Delabie, E.; Jaspers, R.J.E.; von Hellermann, M.G.; Nielsen, Stefan Kragh; Marchuk, O.

\section{Published in:}

Review of Scientific Instruments

Link to article, DOI:

$10.1063 / 1.2955575$

Publication date:

2008

Document Version

Publisher's PDF, also known as Version of record

Link back to DTU Orbit

Citation (APA):

Delabie, E., Jaspers, R. J. E., von Hellermann, M. G., Nielsen, S. K., \& Marchuk, O. (2008). Charge exchange spectroscopy as a fast ion diagnostic on TEXTOR. Review of Scientific Instruments, 79(10), $10 \mathrm{E} 522$.

https://doi.org/10.1063/1.2955575

\section{General rights}

Copyright and moral rights for the publications made accessible in the public portal are retained by the authors and/or other copyright owners and it is a condition of accessing publications that users recognise and abide by the legal requirements associated with these rights.

- Users may download and print one copy of any publication from the public portal for the purpose of private study or research.

- You may not further distribute the material or use it for any profit-making activity or commercial gain

- You may freely distribute the URL identifying the publication in the public portal 


\title{
Charge exchange spectroscopy as a fast ion diagnostic on TEXTOR ${ }^{a)}$
}

\author{
E. Delabie, ${ }^{1, b)}$ R. J. E. Jaspers, ${ }^{1}$ M. G. von Hellermann, ${ }^{1}$ S. K. Nielsen, ${ }^{2}$ and O. Marchuk ${ }^{3}$ \\ ${ }^{1}$ FOM-Rijnhuizen, EURATOM-FOM, NL-3430 BE Nieuwegein, The Netherlands \\ ${ }^{2}$ Association EURATOM-Ris $\phi$ National Laboratory for Sustainable Energy, Technical University of Denmark, \\ DK-4000 Roskilde, Denmark \\ ${ }^{3}$ Forschungszentrum Jülich, EURATOM-FZJ, D-52424 Jülich, Germany
}

(Presented 12 May 2008; received 9 May 2008; accepted 2 June 2008; published online 31 October 2008)

An upgraded charge exchange spectroscopy diagnostic has been taken into operation at the TEXTOR tokamak. The angles of the viewing lines with the toroidal magnetic field are close to the pitch angles at birth of fast ions injected by one of the neutral beam injectors. Using another neutral beam for active spectroscopy, injected counter the direction in which fast ions injected by the first beam are circulating, we can simultaneously measure a fast ion tail on the blue wing of the $D_{\alpha}$ spectrum while the beam emission spectrum is Doppler shifted to the red wing. An analysis combining the two parts of the spectrum offers possibilities to improve the accuracy of the absolute (fast) ion density profiles. Fast beam modulation or passive viewing lines cannot be used for background subtraction on this diagnostic setup and therefore the background has to be modeled and fitted to the data together with a spectral model for the slowing down feature. The analysis of the fast ion $D_{\alpha}$ spectrum obtained with the new diagnostic is discussed. (C) 2008 American Institute of Physics. [DOI: 10.1063/1.2955575]

\section{INTRODUCTION}

Tokamak plasmas can contain a substantial ion population with velocities well above the thermal velocity due to external sources or fusion reactions. Confinement of these ions is crucial for sustaining the plasma heating as well as for protection of the inner wall of fusion reactors. Of special concern are instabilities that expell energetic ions from the plasma and that can be triggered by the fast ions themselves. ${ }^{1}$ In order to clarify the fast ion transport mechanisms, the further development of diagnostics that can provide information on radial profiles of confined fast ions is more than desirable.

Charge exchange spectroscopy (CXRS) has shown to be capable of detecting the slowing down spectrum of ${ }^{3} \mathrm{He}$ beam ions injected in $\mathrm{JET}^{2}$ and fusion born helium at TFTR. ${ }^{3}$ Recently the same diagnostic principle has been successfully applied to the notorious deuterium $n=3 \rightarrow 2\left(D_{\alpha}\right)$ CX spectrum at DIII-D. ${ }^{4,5}$

In this paper the fast ion relevant capabilities of the upgraded CXRS diagnostic on TEXTOR are described. The diagnostic setup is aimed at combining the information from the emission following charge exchange between circulating fast ions and neutral particles from a beam and the emission from excited neutral beam particles.

The use of CXRS as a fast ion diagnostic depends on two items: (1) the diagnostic capability to detect the spectrum, described in Secs. II and III, and (2) the careful interpretation of the signal, partially based on modeling-

\footnotetext{
a) Contributed paper, published as part of the Proceedings of the 17th Topical Conference on High-Temperature Plasma Diagnostics, Albuquerque, New Mexico, May 2008.

${ }^{b)}$ Electronic mail: e.delabie@fz-juelich.de.
}

discussed in Sec. IV. Section V contains an outlook with planned improvements to the diagnostic and consistency checks.

\section{INSTRUMENTATION}

The upgraded CXRS diagnostic on TEXTOR $\left(R_{0}\right.$ $\left.=1.75 \mathrm{~m}, a_{0}=0.47 \mathrm{~m}\right)$ collects light along 19 viewing lines spanning a radial range from 1.85 to $2.13 \mathrm{~m}$. The viewing lines intersect one of the heating beams (NBI1) at local pitch angles with the toroidal magnetic field between $30^{\circ}$ and $40^{\circ}$. These angles are nearly the same as the pitch angles at birth of ions injected by a second neutral beam (NBI2), injected counter the direction of NBI1 at the other side of the torus. This viewing geometry allows us to see the slowing down spectrum of NBI2 ions on the blue wing of the $D_{\alpha}$ spectrum while the beam emission spectrum (BES) is measured on the red wing of the spectrum.

The collected light is guided through optical fibers to a high etendue, high resolution spectrometer $(F / 3, A \Omega=6.2$ $\times 10^{-3} \mathrm{~mm}^{2}$ sr per radial channel, $f=480 \mathrm{~mm}$, dispersion $=4.6 \AA / \mathrm{mm}$ ). The Echelle grating is used in 13th order and a bandpass filter $\left(\lambda_{0}=6561 \AA, \Delta \lambda=200 \AA\right)$ blocks light outside the relevant wavelength range that could overlap with the $D_{\alpha}$ spectrum at different orders. The slit of the spectrometer has been set equal to the width of the image of the fibers in order to maximize the etendue whereas the instrument function can still be satisfactory modeled with a sum of 3 Gaussians with total full width at half maximum of only $1.4 \AA$ A.

The spectra are read out with the low noise (11.6 $e^{-}$rms), high quantum efficiency $(\mathrm{QE}=90 \%)$ PIXIS400B chargecoupled device (CCD) camera from Princeton Instruments. This CCD has a large chip, which allows to record a spectral 


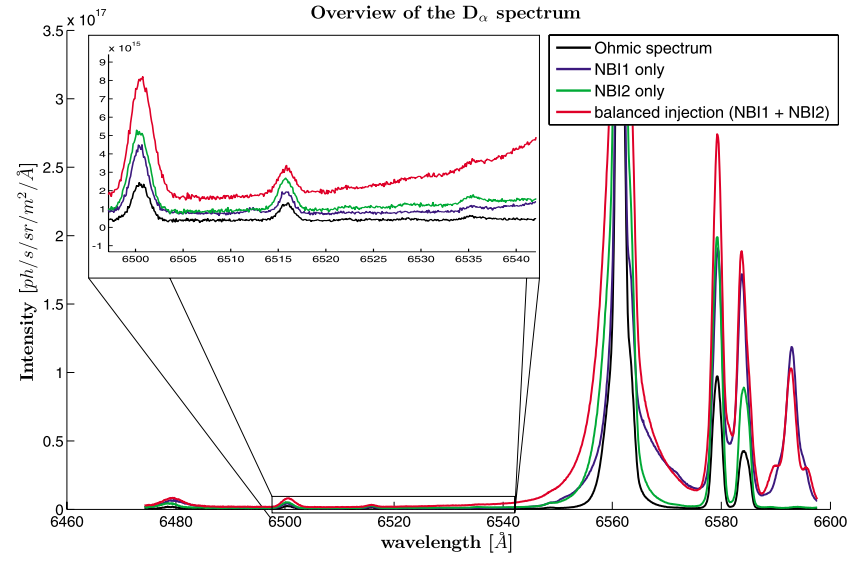

FIG. 1. (Color online) The full $D_{\alpha}$ spectrum is shown (black) for an Ohmic plasma, (blue) a plasma with only the probing beam (no fast ions moving towards the observer), (green) a plasma during injection of NBI2 with ions moving to the observer but without active CX signal, and (red) with both beams. In the inlay the signal in the fast ion relevant wavelength range is shown. A part of the BES (from 658 to $660 \mathrm{~nm}$, overlapping with a CII multiplet) is seen on the red wing of the $D_{\alpha}$ line $(656 \mathrm{~nm})$.

range of $120 \AA$. A mechanical shutter is used during the readout period of the camera, which limits the time resolution to $80 \mathrm{~ms}$ with an effective exposure time of $40 \mathrm{~ms}$. The intense $D_{\alpha}$ line $(656 \mathrm{~nm})$ from excitation of cold atoms near the wall can saturate the detector, but illumation with intense lines from a neon gas discharge lamp show that the charge leakage between pixels on the CCD is negligible.

The fiberbundle can be removed from the periscope, which guides it into the vessel without breaking the vacuum in order to routinely perform a calibration between radial channels and along the length of the CCD chip, necessary for the quantitative study of broad spectral features. These calibrations are performed with an integrating sphere calibration lamp yielding an optical transmission of $8.5 \%$ (assuming the calculated value from the etendue of the spectrometer is correct). During an opening of the vacuum chamber, the last mirror, vacuum window, and periscope optics have been separately calibrated giving a transmission of $32 \%$.

\section{SAMPLE DATA}

In Fig. 1 three spectra are shown: an Ohmic reference spectrum, a spectrum during injection of NBI1 only, and a spectrum during simultaneous injection of NBI1 (counter-Ip) and NBI2 (co-Ip). The deuterium slowing down spectrum (in inlay) has usually a similar or lower intensity than the bremsstrahlung on TEXTOR. Furthermore, the active CXRS slowing down spectrum is contaminated by impurity lines from low ionization stages of mainly $\mathrm{C}$ and $\mathrm{O}$. Also passive charge exchange (PCX) between fast ions and thermal neutrals from the wall has been observed. Therefore a nonlocalized fast ion related signal in the energy range $15-50 \mathrm{keV}$ can sometimes be observerd during high power NBI2 heating without NBI1 acting as a probing beam. No $D_{\alpha}$ related signal from neutrals moving towards the observer has so far been seen above $\sim 15 \mathrm{keV}$ during Ohmic shots or when only NBI1 is injected. A possible approach to eliminate the background is fast (much faster than the slowing down time) beam modulation, ${ }^{5}$

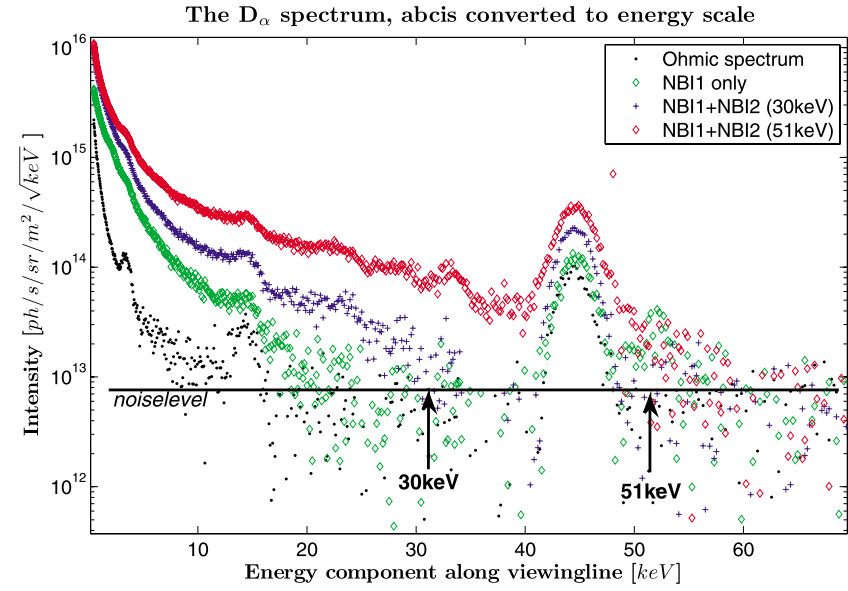

FIG. 2. (Color online) Spectra for different acceleration voltages in NBI2 (bremsstrahlung subtracted). The injection energy is changed from 30 (blue) to $51 \mathrm{keV}$ (red). An Ohmic spectrum (black) and spectrum with only NBI1 (green) are given as reference. Impurity lines overlap with the fast ion spectrum at $15,22,32$, and $45 \mathrm{keV}$.

for technical reasons not achievable due to the long readout time of the camera. An alternative approach is modeling of the background, possible due to the high signal-to-noise ratio of the TEXTOR CXRS spectra and the good spectral resolution, which allows us to resolve the parasitic impurity lines. In Fig. 2 a similar set of spectra is plotted on an energy abcis (the bremsstrahlung, taken equal to the continuum at energies much larger than the beam injection energy, is subtracted). When the NBI2 injection energy is changed from 30 (blue crosses) to $51 \mathrm{keV}$ (red diamonds), the change of the "step" in the spectrum (the onset of the $D_{\alpha}$ slowing down feature at the injection energy) can easily be seen.

\section{MODELING, INTERPRETATION, AND DISCUSSION}

The modeling aspects needed to extract the physical relevant data cover both the modeling of the passive spectra (bremsstrahlung, impurity lines, and possibly PCX) as well as the modeling of the active fast ion spectrum itself. In order to extract the necessary information on all the impurity lines, first an averaged passive spectrum (from the same shot) is fitted. Then the positions and widths of the low intensity lines are taken as initial values or fixed throughout the active beam phase of the discharge (although not all the impurity lines are identified, we assume no active charge exchange lines or emission from high ionisation stages from the rotating core plasma). All parameters concerning the brighter lines can be left free. PCX is neglected.

In a second step a Fokker-Planck (FP) model ${ }^{6}$ is used to derive the classical two dimensional velocity distribution function for neutral beam injected ions (see Fig. 3), with a normalized fast ion source rate. This velocity distribution function is then convoluted with the velocity dependent charge exchange emission rates ${ }^{2,7}$ and projected on a line of sight to give the expected spectral slowing down feature. This spectrum is in good agreement with the experimental data (Fig. 4). It is worth to note that up to now, no significant distortion of the projected velocity distribution with regard to this model has been observed; therefore, the density of the 


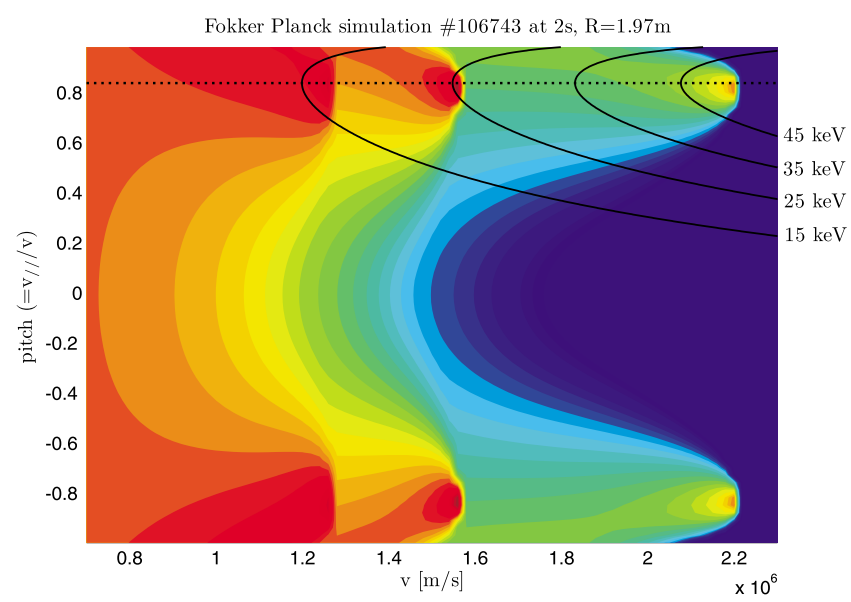

FIG. 3. (Color online) FP simulation (Ref. 6) for a TEXTOR pulse with both beams injected. The beams are identical but the pitch has opposite sign. The dashed line indicates the pitch of a CXRS line of sight. The full curves enclose the area of velocity space that can contribute to the measured signal at specified energies. Within each of these regions the weighting factors are inhomogeneous (see Refs. 2 and 5).

slowing down distribution of each of the energy components in the beam is the only parameter that is retained in the model, which is used to fit to the data.

In a third step, in order to obtain quantitative information on fast ion density profiles, diagnostic artifacts concerning attenuation of the probing neutral beam, uncertainties in the absolute calibration, and alignment with the beam are all taken into account by fitting the BES on the red wing of the $D_{\alpha}$ spectrum. The BES intensities $\left(I_{k}^{\mathrm{BES}}\right)$ directly give the neutral beam $(\mathrm{NB})$ densities integrated along a line of sight. ${ }^{8}$ The slowing down feature, which is then fitted against the data, thus takes the form of Eq. (1). The NB source rates $\left(S_{j}\right)$ are the free parameters and should here be interpreted as source rates plus or minus direct sinks or transport effects in each of the relevant energy regions. $f_{k, j}^{\mathrm{FP}}(\lambda)$ is the charge exchange spectrum (from the FP calculation) between the fast ion population labeled $j$ and probing neutrals with energy corresponding to the index $k . n_{e}$ is the electron density and

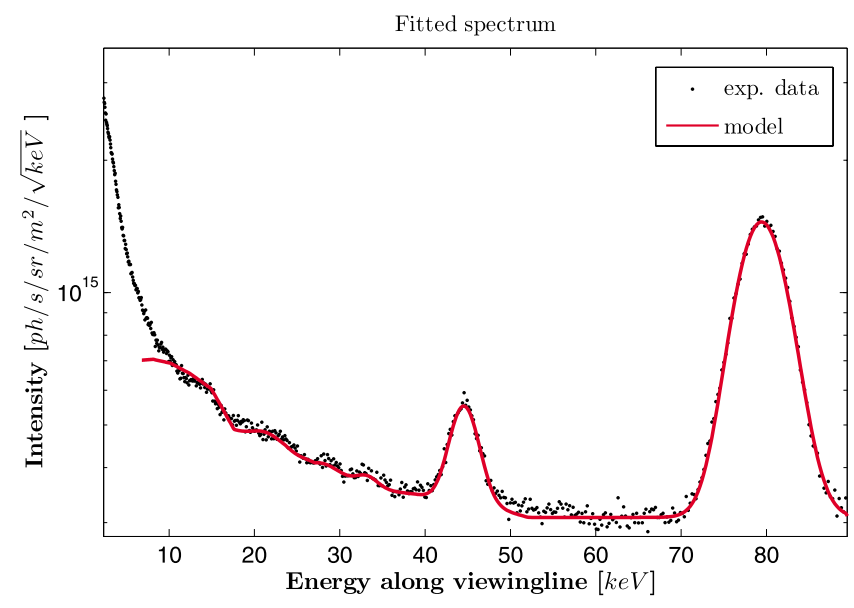

FIG. 4. (Color online) Fitted spectrum. In order to determine the intensity of the slowing down spectrum, the impurity lines and bremsstrahlung must be fitted simultaneously with the synthetic slowing down spectrum from the FP model. Wavelength abcis is converted to energy scale. The "bumps" seen in the $D_{\alpha}$ slowing down tail are impurity lines.
$Q_{k}^{\mathrm{BES}}$ is the effective BES emission rate. ${ }^{7}$ The first summation is over the energy components in the fast ion fueling beam (NBI2) and the second over the energy components in the probing beam (NBI1).

$$
I(\lambda)=\sum_{j=E, E / 2, E / 3} S_{j} \sum_{k=E, E / 2, E / 3} \frac{I_{k}^{\mathrm{BES}}}{n_{e} Q_{k}^{\mathrm{BES}}} f_{k, j}^{\mathrm{FP}}(\lambda) .
$$

From Eq. (1) it is clear that the errorbars on the absolute values of the experimental parameters $S_{j}$, besides from the fitting of the background, will mainly be due to uncertainties in the local beam emission rates $Q^{\mathrm{BES}}$ and to a smaller extent due to the charge exchange cross sections (in $f^{\mathrm{FP}}(\lambda)$ ). However, when inserting reasonable error estimates for all the required experimental input and the involved atomic data, we expect that the accuracy that can be achieved this way is higher than when beam attenuation calculations, exact geometry assessments, and an absolute calibration are required.

\section{OUTLOOK AND CONCLUSIONS}

The diagnostic capability of the TEXTOR CXRS diagnostic to detect a fast ion tail in the $D_{\alpha}$ spectrum has been exploited to obtain radial profiles of beam injected ions. The next step is to check on consistency with information from other diagnostics and compare with accurate modeling of the fast ion deposition profiles. This is expected to provide valuable information on the radial transport of fast ions. Planned improvements to the diagnostic mainly focus on the analysis and an increase in the time resolution.

The collective Thomson scattering (CTS) diagnostic on TEXTOR $^{9}$ provides a unique opportunity to check for data consistency. An experiment has been carried out where CTS and CXRS measured the projected fast ion distribution at the same radial positions and FP pitch angles. A first analysis shows good agreement in velocity space when the same FP model is applied to both CXRS and CTS. For more conclusive results a quantitative analysis of the fast ion radial density profiles must be awaited.

For the next experimental campaign on TEXTOR, the transmission of the pericope optics will be improved by replacing the first mirror with a prism, also strongly reducing the sensitivity of the reflection to the polarization of the incoming light. ${ }^{10}$ Additionally to the acquisition of the 19 channel profile with an $80 \mathrm{~ms}$ time resolution, a set of 9 fibers representing a single radial channel at about half the minor radius will be used to fill the entire etendue of a spectrometer that will be read out with full binning on a $5 \mathrm{~ms}$ timescale.

Slowing down spectra have only been explored in a very limited range of plasma paramaters at TEXTOR and an extension of the model applied to the data, including the full CXRS and BES $H_{\alpha} / D_{\alpha}$ spectrum is ongoing. This will not only improve the accuracy contained in the fast ion part of the spectrum, but would also yield the thermal deuterium and hydrogen densities (in a very similar way as described above for the fast ions). The analysis combining CXRS and BES is expected to give valuable feedback on the status of the involved atomic data, ${ }^{7,11}$ indispensable for quantitative active 
spectroscopy on ITER, where regular absolute calibrations are unlikely and beam attenuation calculations will be prone to errors.

\section{ACKNOWLEDGMENTS}

This work, supported by the European Communities under the Contract of Association between EURATOM-FOM, was carried out within the framework of the European Fusion Programme. The views and opinions expressed herein do not necessarily reflect those of the European Commission. We acknowledge the TEXTOR team.

${ }^{1}$ ITER Physics Expert Group on Energetic Particles, Heating and Current Drive, Nucl. Fusion 39, 2471 (1999).

${ }^{2}$ M. G. von Hellermann, W. G. F. Core, J. Frieling, L. D. Horton, R. W. T. König, W. Mandl, and H. P. Summers, Plasma Phys. Controlled Fusion 35,
799 (1993).

${ }^{3}$ G. McKee, R. Fonck, B. Stratton, R. Bell, R. Budny, C. Bush, B. Grek, D. Johnson, H. Park, A. Ramsey et al., Phys. Rev. Lett. 75, 649 (1995).

${ }^{4}$ Y. Luo, W. W. Heidbrink, K. H. Burrell, D. H. Kaplan, and P. Gohil, Rev. Sci. Instrum. 78, 033505 (2007).

${ }^{5}$ W. W. Heidbrink, Y. Luo, K. H. Burrell, R. W. Harvey, R. I. Pinsker, and E. Ruskov, Plasma Phys. Controlled Fusion 49, 1457 (2007).

${ }^{6}$ J. G. Cordey and W. G. F. Core, Phys. Fluids 17, 1626 (1974).

${ }^{7}$ H. P. Summers, The ADAS User Manual, version 2.6, http://adas. phys. strath. ac. uk (2004).

${ }^{8}$ W. Mandl, R. C. Wolf, M. G. von Hellermann, and H. P. Summers, Plasma Phys. Controlled Fusion 35, 1373 (1993).

${ }^{9}$ H. Bindslev, S. K. Nielsen, L. Porte, J. A. Hoekzema, S. B. Korsholm, F. Meo, P. K. Michelsen, S. Michelsen, J. W. Oosterbeek, E. L. Tsakadze et al., Phys. Rev. Lett. 97, 205005 (2006)

${ }^{10}$ R. J. E. Jaspers, M. G. von Hellermann, E. Delabie, W. Biel, O. Marchuk, and L. Yao, Rev. Sci. Instrum. 79, 10F526 (2008).

${ }^{11}$ O. Marchuk, G. Bertschinger, W. Biel, E. Delabie, M. G. von Hellermann, R. Jaspers, and D. Reiter, Rev. Sci. Instrum. 79, 10F532 (2008). 